\title{
Leishmaniose Tegumentar Americana no Estado de São Paulo: Situação Epidemiológica 2001 - 2002
}

\author{
Vera Lucia Fonseca de Camargo-Neves ${ }^{1}$ e Mitie Tada L.R.F Brasil ${ }^{2}$
}

No Estado de São Paulo a Leishmaniose Tegumentar Americana (LTA) foi descrita pela primeira vez no final do século passado com ocorrência de grande número de casos, porém essas áreas estavam restritas às regiões Oeste e Noroeste do estado, onde o agente identificado foi Leishmania leishmania braziliensis; durante várias décadas a doença perdeu sua importância, porém na década de 70 sua ocorrência foi assinalada no sul do Estado, região considerada anteriormente indene. Nestas áreas, têm sido identificadas manifestações endêmicas e a transmissão humana vem sendo detectada fora do ambiente florestal, ocorrendo nas zonas rural e periurbana. Neste ambiente, a transmissão ocorre no intra, mas principalmente, no peridomicílio (Camargo-Neves 1999). A transmissão no Estado de São Paulo é caracterizada pela ocorrência de casos esporádicos e surtos epidêmicos são característicos de algumas regiões como o Vale do Ribeira, Campinas e Sorocaba, geralmente ligados à ocupação do solo por novas áreas de plantio ou invasão de mata por extensão urbana (Camargo-Neves 1999, Gomes e Camargo-Neves 1998).

Para a análise da transmissão da LTA no Estado de São Paulo, em 2001 e 2002, utilizou-se como fonte de dados: as fichas de notificação de casos encaminhadas pelos municípios aos Serviços Regionais da Superintendência de Controle de Endemias - SUCEN, quando da suspeita de autoctonia, para que se procedesse a investigação entomológica e o controle de foco se necessário e, a base de dados do Sistema Nacional de Agravos de Notificação - SINAN. Das fichas de notificação foram selecionadas as seguintes variáveis: município provável de infecção, número de localidades, sexo, faixa etária e atividade relacionada com o momento da transmissão. Da base de dados do SINAN foram selecionadas as variáveis: forma clínica da doença, motivo da alta e tipo de

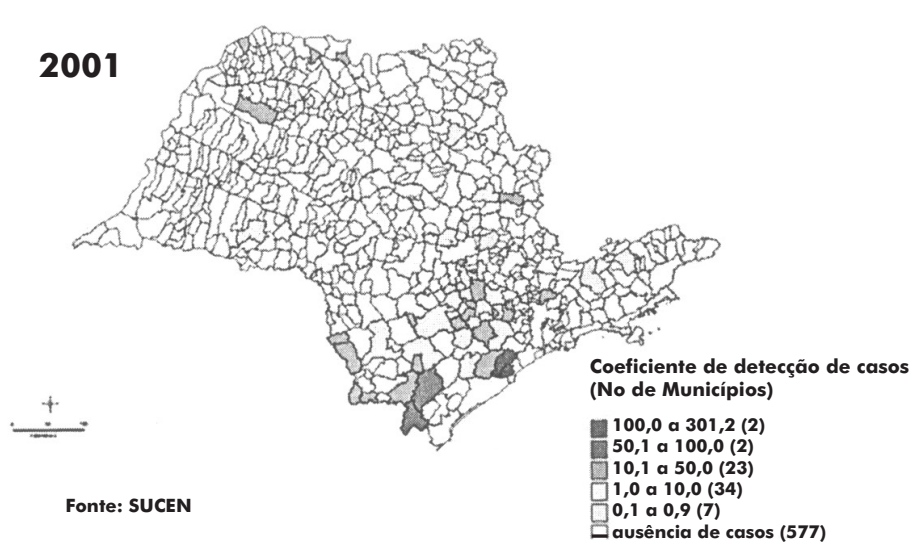

diagnóstico. Com relação às espécies de flebotomíneos relacionados com os focos de transmissão utilizou-se a base de dados de pesquisas entomológicas realizadas pela SUCEN.

Como resultados, em 2001, foram detectados pelo sistema de vigilância epidemiológica 307 (82,7\%) casos autóctones de ITA, destes foram notificados à SUCEN 254 casos distribuídos por 68 municípios paulistas, envolvendo, pelo menos, 149 localidades (Figura 1). Em 2002, verificou-se um aumento no número de casos e do número de municípios com transmissão quando comparados ao ano anterior, neste ano foram registrados no SINAN, 564 casos distribuídos em 146 municípios paulistas, destes foram notificados $521(92,4 \%)$ casos aos serviços regionais da SUCEN, distribuídos por 106 municípios paulistas ocorrendo em pelo menos 327 localidades (Figura 1 e 2), configurando que a transmissão da ITA, no período estudado, continua se dando de forma esporádica, com já observado anteriormente. Em estudo realizado por Katz (1997), sobre a epidemiologia da LTA no Estado de São Paulo, no período de 1986 a 1995, foi observado que, apesar da expansão das áreas de transmissão no Estado, nem todos os municípios apresentaram surtos da doença, caracterizando-se na maioria dos municípios como uma doença de registro de casos esporádicos e de baixa incidência.

Os coeficientes de detecção de casos, por região administrativa da SUCEN, variaram entre de 0,37 casos / 100.000 habitantes (região de São José do Rio Preto) e 102,70 casos / 100.000 hab (Vale do Ribeira), em 2001 e 0,89 casos/100.000 hab. (Litoral Norte) e 158,03 casos/100.000 hab (Vale do Ribeira), em 2002 (Figura 1). Chama atenção o aumento dos coeficientes de detecção na maioria das regiões do Estado (Figuras 1 a 3). Observou-se em 2002 a expansão da doença, sendo que o maior número de casos foram registrados nas regiões do Vale do Ribeira, Sorocaba e Campinas.

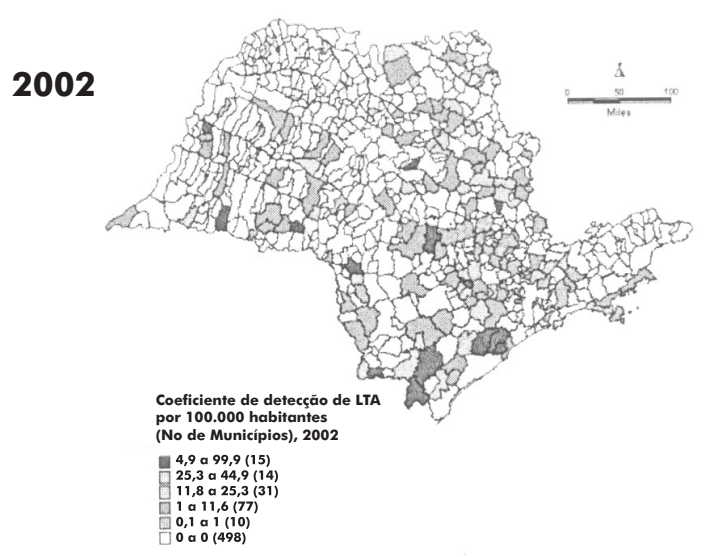

Figura 1 - Distribuição dos coeficientes de detecção de casos de Leishmaniose Tegumentar Americana. Estado de São Paulo, 2001 - 2002. 


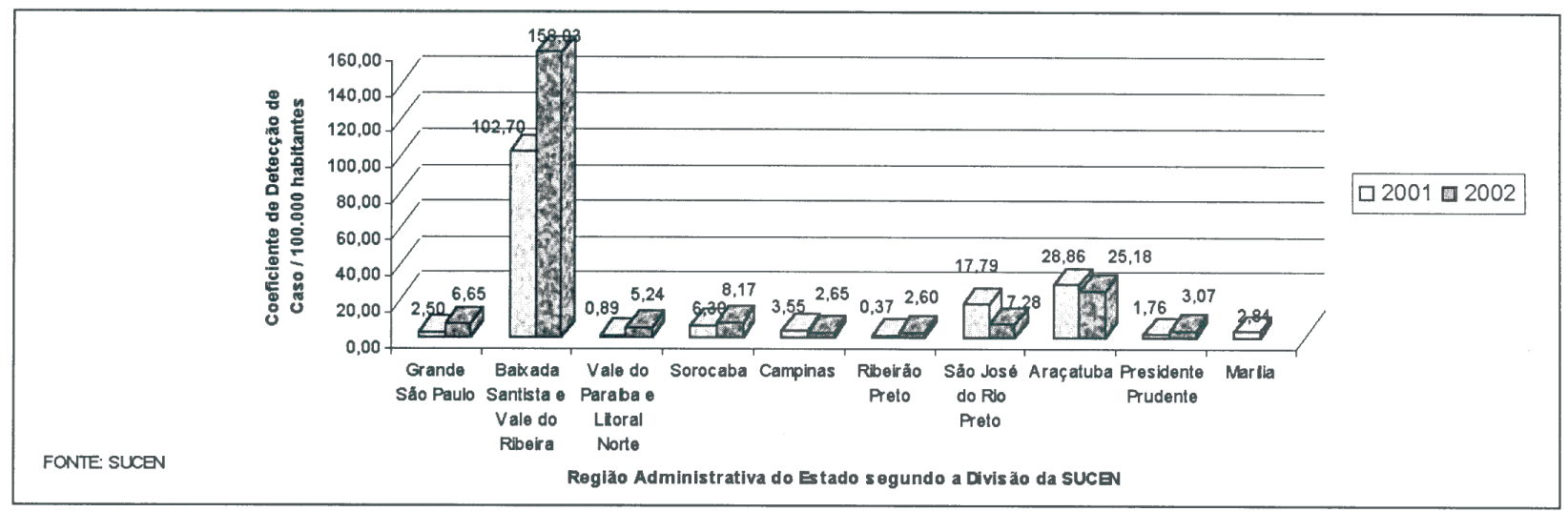

Figura 2 - Coeficiente de detecção de caso de Leishmaniose Tegumentar Americana, segundo região administrativa do Estado de São Paulo, 2001 e 2002.

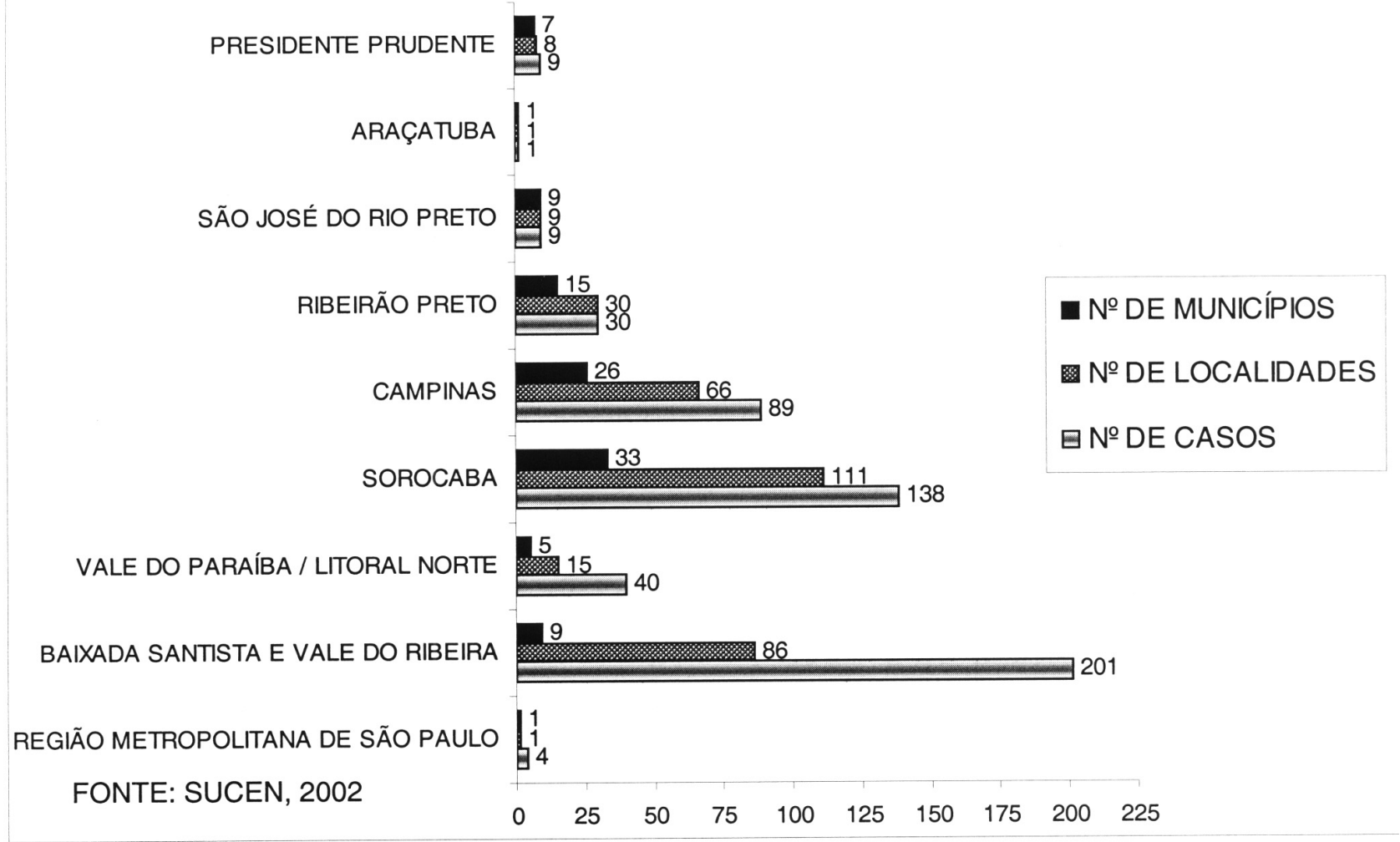

Figura 3 - Distribuição do número de casos, de municípios e de localidades de Leishmaniose Tegumentar Americana, segundo região administrativa do Estado de São Paulo, 2002.

A transmissão foi predominante para o sexo masculino e faixa etária de 21 anos de idade e mais nos dois anos estudados (Figura 4 e Tabelas 1 e 2).

Verificou-se, em 2001, que em $52,0 \%$ dos casos a transmissão esteve relacionada com atividades envolvendo 0 ambiente domiciliar e cerca de $10,0 \%$ dos casos relataram atividades de lazer, principalmente, na região de Campinas (Tabela 3). Destacando-se a região do Vale do Ribeira onde a transmissão ocorreu no ambiente domiciliar, acometendo todas as faixas etárias, sendo que em $18,9 \%$ dos casos, eram crianças na faixa etária de 2 a 10 anos de idade (Tabela 1).
Em 2002, a investigação de foco ocorreu em apenas 38,2\% dos casos notificados à SUCEN. Verificou-se que atividade de lazer esteve relacionada com a transmissão, principalmente, na região de Sorocaba. 0 alto percentual de ignorados deve-se a falta de investigação dos casos ocorridos no Litoral Sul e Vale do Ribeira, embora se admita que o padrão de transmissão nesta região seja o mesmo, isto é a transmissão envolve atividades desenvolvidas no ambiente domiciliar. Nesta região merece destaque 0 município de Itariri pela ocorrência de surtos importantes nos anos mencionados e, onde a transmissão domiciliar envolveu a maioria dos casos. Destaca-se ainda a 

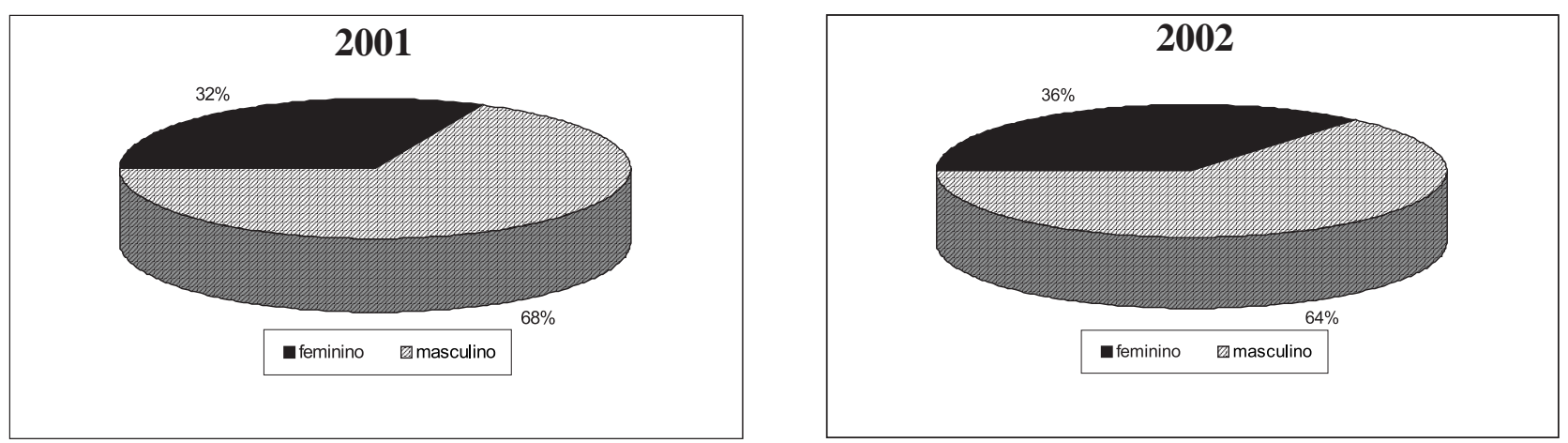

\section{Fonte: SUCEN}

Figura 4 - Distribuição percentual de casos de Leishmaniose Tegumentar Americana segundo sexo. Estado de São Paulo, 2001 - 2002.

Tabela 1- Distribuição de casos de Leishmaniose Tegumentar Americana por faixa etária e região administrativa da SUCEN (Serviço Regional). Estado de São Paulo, 2001.

\begin{tabular}{|c|c|c|c|c|c|c|c|c|c|c|c|}
\hline \multirow{3}{*}{ Serviço Regional } & \multicolumn{10}{|c|}{ Faixa Etária } & \multirow{3}{*}{$\begin{array}{r}\text { Total } \\
\mathrm{n}^{\circ}\end{array}$} \\
\hline & \multicolumn{2}{|c|}{$<=1$} & \multicolumn{2}{|c|}{2 a 10} & \multicolumn{2}{|c|}{11 a 20} & \multicolumn{2}{|c|}{$21 \mathrm{E}+$} & \multicolumn{2}{|c|}{ Ignorada } & \\
\hline & $\mathrm{n}^{0}$ & $\%$ & $\mathrm{n}^{0}$ & $\%$ & $\mathrm{n}^{0}$ & $\%$ & $\mathrm{n}^{0}$ & $\%$ & $\mathrm{n}^{0}$ & $\%$ & \\
\hline 1. Grande São Paulo & 1 & 7,7 & 1 & 7,7 & 3 & 23,1 & 8 & 61,5 & 0 & 0,0 & 13 \\
\hline 2. Baixada Santista e Vale do Ribeira & 1 & 1,1 & 18 & 18,9 & 18 & 18,9 & 57 & 60,0 & 1 & 1,1 & 95 \\
\hline 3. Taubaté e Litoral Norte & 0 & 0,0 & 1 & 14,3 & 2 & 28,6 & 0 & 0,0 & 4 & 57,1 & 7 \\
\hline 4. Sorocaba & 0 & 0,0 & 5 & 5,1 & 15 & 15,2 & 77 & 77,8 & 2 & 2,0 & 99 \\
\hline 5. Campinas & 0 & 0,0 & 0 & 0,0 & 2 & 8,0 & 22 & 88,0 & 1 & 4,0 & 25 \\
\hline 6. Ribeirão Preto & 0 & 0,0 & 0 & 0,0 & 0 & 0,0 & 2 & 100,0 & 0 & 0,0 & 2 \\
\hline 8. São Jose do Rio Preto & 0 & 0,0 & 0 & 0,0 & 0 & 0,0 & 4 & 100,0 & 0 & 0,0 & 4 \\
\hline 9. Araçatuba & 0 & 0,0 & 0 & 0,0 & 0 & 0,0 & 2 & 100,0 & 0 & 0,0 & 2 \\
\hline 10. Presidente Prudente & 0 & 0,0 & 0 & 0,0 & 0 & 0,0 & 4 & 100,0 & 0 & 0,0 & 4 \\
\hline 11. Marília & 0 & 0,0 & 0 & 0,0 & 0 & 0,0 & 3 & 100,0 & 0 & 0,0 & 3 \\
\hline Total & 2 & 0,8 & 25 & 9,8 & 40 & 15,7 & 179 & 70,5 & 8 & 3,1 & 254 \\
\hline
\end{tabular}

Fonte: SUCEN

Tabela 2 - Distribuição de casos de Leishmaniose Tegumentar Americana por faixa etária e região administrativa da SUCEN (Serviço Regional). Estado de São Paulo, 2002.

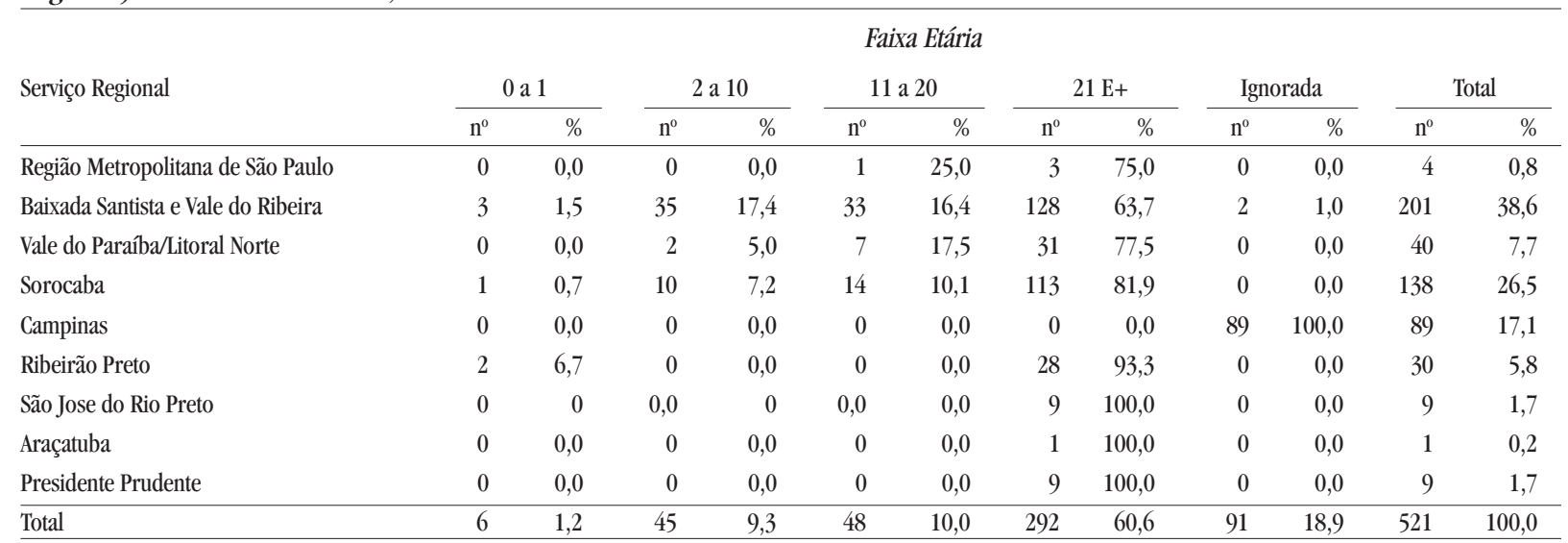

Fonte: SUCEN

região do Litoral Norte, em que a atividade relacionada com a transmissão envolveu o ambiente domiciliar, devido principalmente à ocupação do solo para moradia (Tabela 4). No período estudado pôde-se observar que, nas regiões central e noroeste do Estado, a transmissão esteve relacionada às atividades de lazer, onde os rios são os principais atrativos, constituindo um ecótopo importante na transmissão da LTA dada a presença de matas ciliares, o que explicaria o alto percentual de indivíduos maiores de 20 anos de idade, nestas regiões.
A forma clínica mais comum diagnosticada foi a cutânea, tendo sido registrada em 73,0\% (2001) e 87,8\% (2002) dos indivíduos (Figura 5), mostrando uma melhoria no sistema de vigilância e indicando que a maioria dos casos foram diagnosticados precocemente. As lesões de mucosa registradas em 2001, ocorreram em 64,8\% dos indivíduos com provável local de transmissão na região do Vale do Ribeira. No entanto, em 2002, a forma cutânea mucosa foi verificada em $11,2 \%$ dos casos notificados, provenientes das 
Tabela 3 - Distribuição de casos de Leishmaniose Tegumentar Americana por atividade relacionada no momento da transmissão e região administrativa da SUCEN (Serviço Regional). Estado de São Paulo, 2001.

\begin{tabular}{|c|c|c|c|c|c|c|c|c|c|}
\hline \multirow{3}{*}{ Serviço Regional } & \multicolumn{8}{|c|}{ Atividade relacionada com a transmissão } & \multirow{3}{*}{$\begin{array}{r}\text { Total } \\
\mathrm{n}^{0} \\
\end{array}$} \\
\hline & \multicolumn{2}{|c|}{ Domiciliar } & \multicolumn{2}{|c|}{ Lazer } & \multicolumn{2}{|c|}{ Profissional } & \multicolumn{2}{|c|}{ Ignorada } & \\
\hline & $\mathrm{n}^{0}$ & $\%$ & $\mathrm{n}^{0}$ & $\%$ & $\mathrm{n}^{0}$ & $\%$ & $\mathrm{n}^{0}$ & $\%$ & \\
\hline 1. Grande São Paulo & 2 & 15,4 & 1 & 7,7 & 0 & 0,0 & 10 & 76,9 & 13 \\
\hline 2. Baixada Santista e Vale do Ribeira & 95 & 100,0 & 0 & 0,0 & 0 & 0,0 & 0 & 0,0 & 95 \\
\hline 3. Taubaté e Litoral Norte & 4 & 57,1 & 1 & 14,3 & 0 & 0,0 & 2 & 28,6 & 7 \\
\hline 4. Sorocaba & 25 & 25,3 & 0 & 0,0 & 17 & 17,2 & 57 & 57,6 & 99 \\
\hline 5. Campinas & 5 & 20,0 & 17 & 68,0 & 1 & 4,0 & 2 & 8,0 & 25 \\
\hline 6. Ribeirão Preto & 0 & 0,0 & 1 & 50,0 & 0 & 0,0 & 1 & 50,0 & 2 \\
\hline 8. São Jose do Rio Preto & 0 & 0,0 & 4 & 100,0 & 0 & 0,0 & 0 & 0,0 & 4 \\
\hline 9. Araçatuba & 0 & 0,0 & 2 & 100,0 & 0 & 0,0 & 0 & 0,0 & 2 \\
\hline 10. Presidente Prudente & 0 & 0,0 & 0 & 0,0 & 0 & 0,0 & 4 & 100,0 & 4 \\
\hline 11. Marília & 1 & 33,3 & 0 & 0,0 & 2 & 66,7 & 0 & 0,0 & 3 \\
\hline Total & 132 & 52,0 & 26 & 10,2 & 20 & 7,9 & 76 & 29,9 & 254 \\
\hline
\end{tabular}

Fonte: SUCEN

Tabela 4 - Distribuição de casos de Leishmaniose Tegumentar Americana por atividade relacionada no momento da transmissão e região administrativa da SUCEN (Serviço Regional). Estado de São Paulo, 2002.

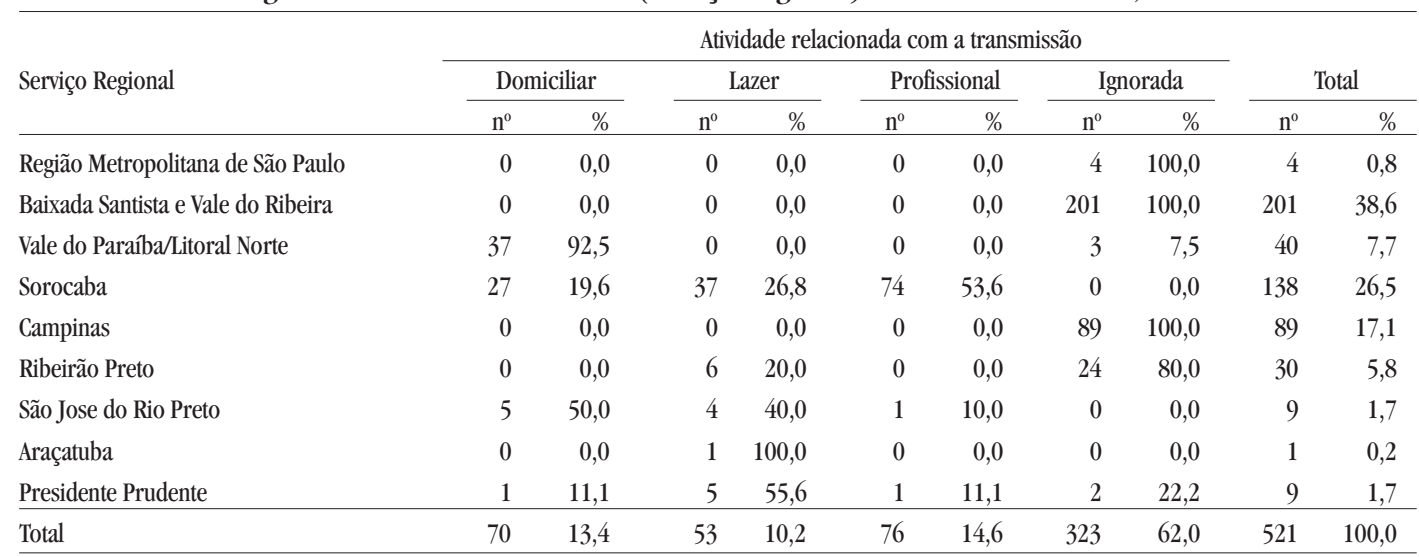

Fonte: SUCEN

regiões de Campinas (2,7\%); de Marília (2,1\%) e Ribeirão Preto $(1,8 \%)$ e os demais distribuídos pelas outras regiões do Estado. Cabe ressaltar, nesse ano, o alto percentual de casos investigados sem informação quanto à forma clínica (Figura 5).

Verificou-se uma melhora no critério diagnóstico, na grande maioria dos casos o diagnóstico se deu por meio de exames complementares. Em 2001, 51,0\% dos casos foram confirmados apenas pelo critério clínico, enquanto em 2002, 87,0\% deles foram confirmados por critério clínico laboratorial. Verificouse redução do número de casos que evoluíram para cura $(65,0 \%)$, quando comparado com o ano anterior (94,0\%, em 2001), este resultado pode ser explicado, em grande parte, ao preenchimento inadequado da ficha de investigação e alimentação do sistema (Figura 5).

Com relação à investigação de suspeitos de efeitos adversos causados pelo antimoniato de N-metil glucamina (Glucantime ${ }^{\circledR}$ ), droga de escolha para o tratamento da LTA, em 2001 foram investigados cinco casos, sendo que quatro deles notificados pelo Instituto de Infectologia Emilio Ribas -SES/SP e um caso pela Regional de Saúde de Marília. Tratavam-se de pacientes acima de 50 anos e que apresentaram quadros como pancreatite tóxica, hepatite tóxica e outros que não puderam ser classificados pela variedade de manifestações clínicas. Verificou-se clara dificuldade em relacionar estas ocorrências diretamente ao uso do medicamento, podendo também ter associação com alguma condição prévia do paciente e não detectado pelos exames de laboratório realizados antes do tratamento. Estas ocorrências alertaram para o monitoramento mais criterioso do tratamento e, também, para a importância da notificação de casos suspeitos de efeitos adversos à droga.

A priorização de outras atividades em detrimento da investigação e controle de foco de LTA poderia explicar o aumento do número de casos em relação ao ano anterior, porém sabendose do caráter sazonal desta endemia e pelo grande número de localidades com a ocorrência apenas de um caso, talvez este aumento não possa ser explicado pela falta de atividade de controle na maioria das regiões, principalmente naqueles em que a atividade de lazer foi a principal atividade relacionada com a transmissão. Sabe-se também, que projetos de ocupação do solo, para moradia, vêm a ser um dos fatores para a ocorrência da LTA principalmente na região do Litoral Norte indicando a necessidade de implementar um programa de capacitação para médicos e outros profissionais de saúde, para diagnóstico e tratamento precoce. Por outro lado, no Litoral Sul e Vale do Ribeira, áreas de colonização antiga, outros fatores ambientais, como também a presença de animais sinantrópicos 

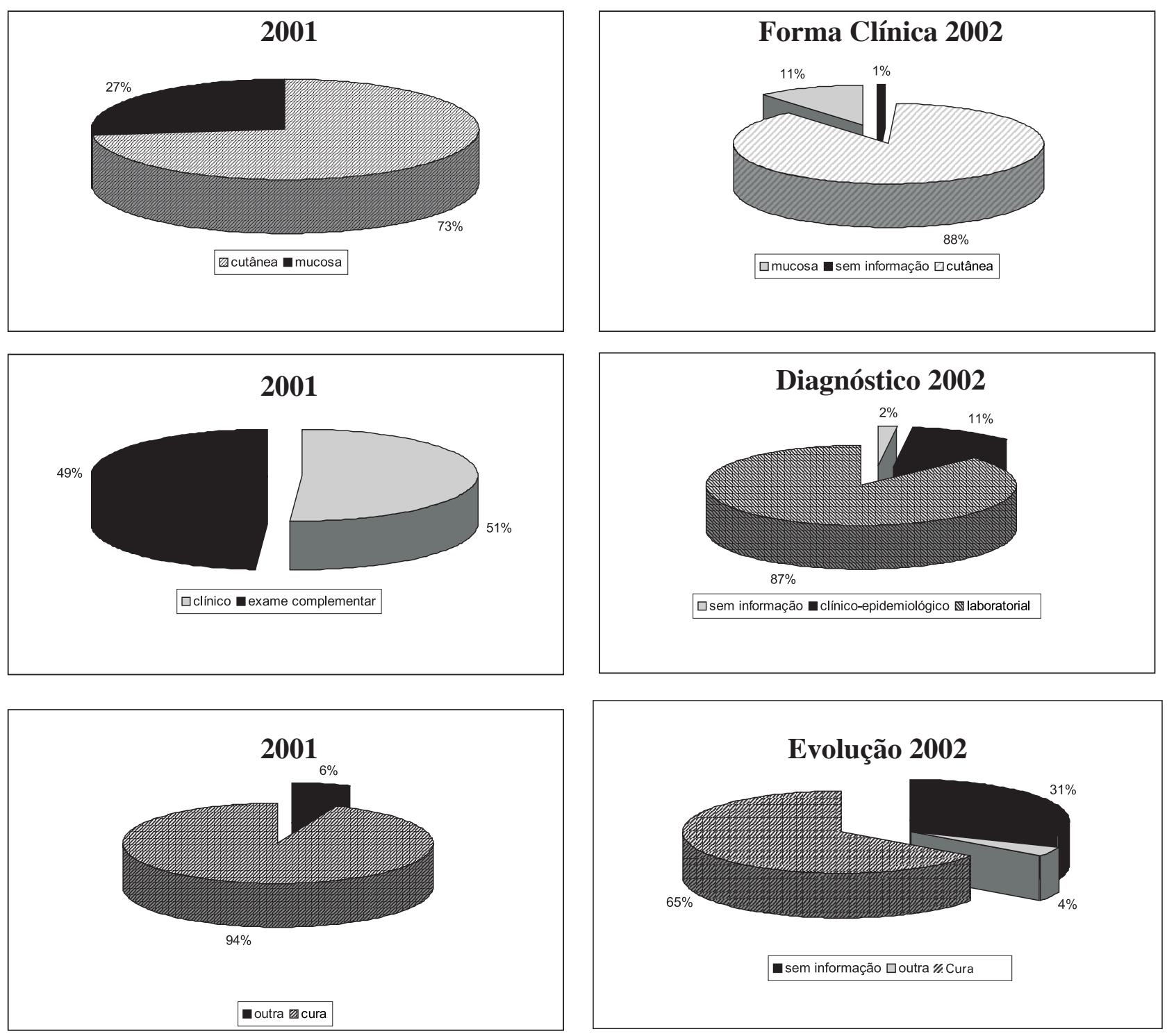

Figura 5 - Distribuição percentual de casos de Leishmaniose Tegumentar Americana notificados no sistema nacional de agravos de notificação - SINAN, segundo a forma clínica, critério diagnóstico e evolução. Estado de São Paulo, 2001 - 2002.

e roedores silvestres próximos às residências, devido à proximidade destas com a mata, devem estar relacionados com aumento do número de casos, ocorrendo no ambiente domiciliar.

Com relação à espécie de flebotomíneo, Lutzomyia intermedia s.l. esteve presente em $90 \%$ dos focos investigados, em 2001. Camargo-Neves e cols. (2001) estudando a distribuição das espécies de flebotomíneos em focos de transmissão da LTA, no período de 1986 a 1995, demonstraram que os municípios que apresentaram as maiores médias dos coeficientes padronizados de incidência estavam também presentes as espécies L. migonei, $L$. fischeri e $L$. intermedia s.l. Sendo que a primeira espécie teria um papel preponderante no ambiente extra-domiciliar e a última no ambiente domiciliar. Estes municípios estão situados na região geomorfológica do Planalto Atlântico e da Província Costeira, que vêm a ser as regiões onde se concentraram o maior número de casos detectados em 2001 e 2002.

A crescente notificação de Leishmaniose Tegumentar Americana no Estado de São Paulo requer explicações para o esclarecimento de como essa doença vem ocorrendo, uma vez que o perfil epidemiológico da transmissão vem sendo modificado de acordo com crescimento sócio-econômico, promovendo modificação do perfil das zonas rurais e expansão dos centros urbanos.

Apesar de ainda apresentar deficiências nas articulações interinstitucionais vigilância epidemiológica, controle do vetor e laboratório, tanto na esfera estadual como na municipal, o sistema de vigilância epidemiológica apresentou uma melhora, no período estudado, embora ainda ocorra a priorização de outras atividades em detrimento da investigação e controle de foco de LTA. No entanto, deve-se também considerar, que o sucesso da atividade de controle está associada à agilidade na identificação de focos da doença pelos serviços de vigilância, dos recursos humanos disponíveis, para a realização da captura de flebotomíneo na época mais propícia. Por outro lado, o atraso na identificação destes focos está associado ao longo período de incubação da doença, ao retardamento do paciente à procura 
pela assistência médica e o tempo de obtenção do diagnóstico laboratorial, como já apontado anteriormente por Gomes \& Camargo-Neves (1998).

\section{REFERÊNCIAS BIBLIOGRÁFICAS}

Brasi, MTLR - Relatório de investigação dos casos suspeitos de efeitos adversos causados pelo uso de Glucantime(Antimoniatode N-Metil Glucamina) no tratamento de leishmaniose tegumentar Americana - CVE- SES-SP , 2001

Camargo-Neves VLF. Características da Transmissão da Leishmaniose Tegumentar Americana no Estado de São Paulo, Brasil. Dissertação de Mestrado, Faculdade de Saúde Pública da Universidade de São Paulo, São Paulo, SP, 1999.
Camargo-Neves VLF, Gomes AC, Antunes JLF. Correlação da presença de espécies de flebotomíneos (Diptera: Psychodidae) com registros de casos da leishmaniose tegumentar americana no Estado de São Paulo, Brasil. Revista da Sociedade Brasileira de Medicina Tropical 35: 299-306, 2002.

Gomes, A C \& Camargo-Neves, VLF "Estratégias e perspectivas de controle da Leishmaniose Tegumentar Americana no Estado de São Paulo". Revista da Sociedade Brasileira de Medicina Tropical 31 (6): 553-558, 1998.

Katz, G. Epidemiologia da Leishmaniose Tegumentar Americana no Estado de São Paulo. São Paulo, USP. Dissertação de Mestrado, Faculdade de Medicina da Universidade de São Paulo, 1997. 